\title{
The Impact of Chronic Social Stress on Emotional Behavior in Mice and the Therapeutic Effect of Peripheral Mild-Heat Stimulation
}

\author{
Hang Liu1, Takuji Yamaguchi², Kenji Ryotokuji', Satoru Otani',4, Hiroyuki Kobayashi',5, \\ Masako Iseki' ${ }^{1}$ Eichi Inada ${ }^{1}$ \\ ${ }^{1}$ Department of Anesthesiology and Pain Management, School of Medicine, Juntendo University, \\ Tokyo, Japan \\ ${ }^{2}$ Center for Advanced Kampo Medicine and Clinical Research, Juntendo Graduate School of Medicine, \\ Tokyo, Japan \\ ${ }^{3}$ Institute of Transcultural Studies, Ryotokuji University, Chiba, Japan \\ ${ }^{4}$ Center for Medical Education, Ryotokuji University, Chiba, Japan \\ ${ }^{5}$ Department of Hospital Administration, Juntendo University Graduate School of Medicine, \\ Tokyo, Japan \\ Email: liu@juntendo.ac.jp
}

Received 2 September 2015; accepted 16 October 2015; published 19 October 2015

Copyright (C) 2015 by authors and Scientific Research Publishing Inc.

This work is licensed under the Creative Commons Attribution International License (CC BY).

http://creativecommons.org/licenses/by/4.0/

(c) (i) Open Access

\section{Abstract}

In today's stressful society, mental diseases such as adjustment disorder, affective disorders including anxiety and depression, and chronic fatigue syndrome could be attributed to social stress and are considered as serious social problems. It has been long known in the acupuncture and moxibustion therapies in Japan and China that stimulation at Zhongwan and Taichong points have effects on stress-related anxiety and depression. In the present study, we therefore tested the effect of peripheral mild-heat stimulation at Zhongwan and Taichong on anxiety and brain biochemistry using a mouse model of chronic social stress. Male ddY mice (4 weeks old) were stressed by isolated rearing for 6 weeks. They received mild-heat stimulation $\left(43^{\circ} \mathrm{C}\right.$ for $\left.5 \mathrm{~min}\right)$ at Zhongwan and Taichong under isoflurane anesthesia after 4 weeks of the social isolation 3 times a week for 2 weeks, using the "Stress Free Apparatus" (Ryoken Co., Ltd). Control mice received only isoflurane anesthesia. Group-reared mice also underwent the same stimulus/anesthesia sessions. Two days after the last peripheral heat stimulation, anxiety was determined by the light/dark box test as the time spent in the light compartment and the number of transfer between the light and dark compartments. Before and after the last stimulation, blood was collected from an artery at the back of 
the eye, and serum corticosterone, immunoglobulin E (IgE) which was an index of the inflammatory immune system, and interleukin 6 (IL-6) were measured using ELISA Kit. The frontal cortex was collected after the behavioral test, and the dopamine and serotonin contents were measured also by ELISA Kit. A significant decrease of the time spent in the light compartment was observed after the isolated rearing $(p<0.01)$. Mild-heat stimulation significantly increased the time spent in the light compartment, indicating an anti-anxiety effect. Serum corticosterone, IgE and IL-6 were increased after stress, and the amount of serotonin in the frontal cortex was found decreased. The increased corticosterone, IgE and IL- 6 were cancelled by mild-heat stimulation. These results suggest that chronic social isolation has negative biological and emotional impacts in mice and that peripheral mild-heat stimulation alleviates at least part of these stress effects.

\section{Keywords}

\section{Chronic Stress, Anxiety, Acupuncture, Frontal Cortex, Corticosterone}

\section{Introduction}

In our society, stress is known to be related to many clinical conditions. Especially, mental diseases such as adjustment disorder, depression, and chronic fatigue syndrome caused by chronic social stress are recognized as serious social problems [1]. Moreover, mood disorder has been linked to physical diseases for years. For example, endocrine diseases (e.g. hypothyroidism, hyperthyroidism, and Cushing's syndrome), organic brain diseases (e.g. Parkinson's disease, multiple sclerosis, and cerebrovascular disease), and other diseases including cancer, systemic erythematodes (SLE), cardiac infarct, and chronic pain, can be caused by anxiety and depression resulting from social stress [2]. Nevertheless, currently, there are no effective treatments available for these stressrelated diseases.

It has been reported in the acupuncture and moxibustion therapies practiced in Japan and China that Zhongwan and Taichong stimulations have effects on stress-related anxiety and depression [3]-[5]. Zhongwan (CV-12) is the 12th acupuncture point that belongs to the conception vessel meridian. Zhongwan activates the autonomic nervous system [6], and has an anti-inflammatory effect [7] as well as a palliative effect on chronic anxiety disease [8] and severe depression [9] [10]. Taichong (LR-3) is the 3rd acupuncture point of the liver meridian of foot-jueyin, and is a transport point and a yuan-primary point of the foot. Taichong stimulation has been shown to have a palliative effect on epilepsy, insanity, bile, temper, and lowering of mood [3]-[5]. It is also thought that taichong stimulation has an antihypertensive property [11].

Recently, as a sophisticated version of the traditional acupuncture method, the pinpoint plantar low-wavelength infrared light irradiation (PP-LILI), known as stress-free therapy ${ }^{\circledR}$ was introduced (Trademark Registration No.5495960; patent Application No. PCT [Patent Cooperration Theaty]/JP [Japan] 2012/073972). Thus, application of a mild-heat stimulation at a specific plantar part with the PP-LILI was shown to cause a decrease of blood ACTH, an increase of deep body temperature, and an increase of bran blood flow [12]-[14], which are expected to have anti-anxiety effects. This novel method draws attention as a possible therapeutic option to treat stress-related disorders. To evaluate its effects objectively, the basic animal research is now required.

Along with the introduction of the concept of chronic stressor, a syndrome called functional somatic syndrome (FFS) is often mentioned [15] [16]. For example, the developmental environment in the juvenile stage is considered as one of the factors to cause pathogenesis of mental diseases, and rodents reared in isolation for a long time in the juvenile stage serve as important model animals [17] [18]. Rearing small laboratory animals such as mice and rats individually for a long time leads to behavioral changes not seen in group-reared normal animals, particularly called "isolation syndrome" [19] that includes anxiety, depression, increased aggression, and memory disorder [20]. The isolation syndrome is considered as a model for human mental diseases such as schizophrenia, anxiety disorder, mood disorder, and drug dependence [21] [22].

In the present study, behavioral effects of a 6-week isolated rearing in mice starting at the juvenile stage (28 days of age) were quantified. As a therapeutic option for stress-related disorders, we then tested the effect of mild-heat stimulation at Zhongwan and Taichong points (Figure 1), using the PP-LILI. Moreover, blood levels of corticosterone, immuno-globulin E (IgE), interleukin-6 (IL-6) as well as the amount of dopamine and seroto- 


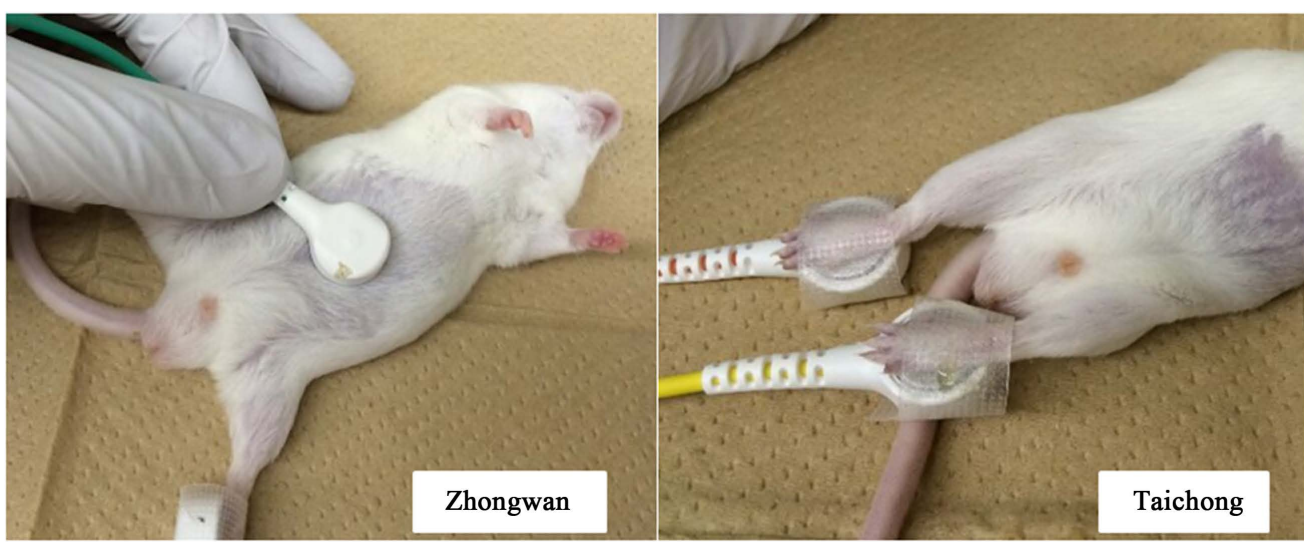

(a)

(b)

Figure 1. The locations of the stimulation to Zhongwan and Taichong points in the mouse are shown. In our condition, heat stimulation was applied to these points as well as areas surrounding these points to some extent.

nin in the frontal cortex were measured to determine whether the PP-LILI also normalizes changes in these biological stress markers.

\section{Material and Method}

\subsection{Animals and Stress Protocol}

Three weeks old male ddY mice were purchased from Japan SLC Co, Ltd. (Shizuoka, Japan). The ddY strain is an outbred one, and has been maintained as a closed colony. Mice of the strain show good reproductive performance and superior growth. In Japan, this strain, along with ICR strain, has been widely used in trials for drug efficacy and various fields of researches, such as pharmacological, phamacokinetic, and toxicological studies [23]. After a week of habituation, animals of the experimental group (stressed group) were placed individually in a transparent cage $(9 \times 13 \times 20 \mathrm{~cm})$ for 6 weeks ("isolation" group). A wooden wall was inserted between the cages so that the isolated animals are deprived of visual contact with others. As the control group, 5 mice were reared together in a cage of the size $23 \times 31 \times 15.5 \mathrm{~cm}$ ("group" group). All animals were reared under the following conditions; $23^{\circ} \mathrm{C} \pm 2^{\circ} \mathrm{C}$, relative humidity $55 \% \pm 10 \%, 12$ hours of light (7:00-) and dark (19:00-) cycle, free drinking, and normal food consumption (MF, Oriental Yeast Co., Ltd., Tokyo, Japan). The experiments were conducted in accordance with the guideline of Juntendo University Medical School Animal Testing (JACUC) (Registration no. 1022).

\subsection{Mild-Heat Stimulation Method}

After 4 weeks of isolated rearing, mild-heat stimulation was applied at Zhongwan (CV-12) and Taichong (LR-3) by using a mild-heat stimulator (Stress Free Apparatus, Ryoken Co, Ltd. Tokyo) under isoflurane anesthesia. Briefly, Zhongwan is located at the midpoint between the bottom edge of the sternumand the navel, and Taichong is at the junction of the 1st and 2nd metatarsals of the foot. However, in our method, areas surrounding these points were also heat-stimulated to some extent (Figure 1). The temperature of stimulation was set at $43^{\circ} \mathrm{C}$, and the duration of stimulation was 5 min/day, which was repeated 3 times a week for 2 weeks. This stimulation parameter is based on the previous human studies [12]-[14], slightly modified to accelerate data acquisition. Control mice received only isoflurance anesthesia. The same operations were performed on the group-reared mice.

\subsection{Light/Dark Box Test Method}

Two days after the last mild-heat stimulation, behavioral changes were evaluated by using a light/dark box. After habituating the mice in the dark compartment of the light/dark box $(20 \times 16 \times 20 \mathrm{~cm})$ for 1 minute, the gate was opened, and the time spent in the light compartment and the number of transfer between two compartments were measured for $5 \mathrm{~min}$ as the indicator of anxiety state. 


\subsection{The Measurement of Blood Serum Component}

After 6 weeks of isolated rearing (in the case of corticosterone measurement, also after 2 and 4 weeks), blood was collected from an artery at the back of the eye, and the blood serum was obtained after centrifugation $\left(15,000 \mathrm{rmp} \times 20\right.$ minutes, $\left.4^{\circ} \mathrm{C}\right)$. The blood serum was frozen and preserved at $-20^{\circ} \mathrm{C}$. Serum corticosterone, IgE, and IL-6 were measured by ELISA Kit: Mouse Corticosterone Kit (Assay Designs, Michigan, USA), Mouse IgE Kit (Morinaga Institute of Biological Science Inc. Yokohama, Japan), and Mouse IL-6 Kit (mouse IL-6 Immunoassay R \& D Systems USA) were used for measuring, respectively, corticosterone, IgE, and IL-6 levels.

\subsection{The Measurement of Brain Monoamines}

Two days after the last stimulation, the frontal cortex was collected, frozen and preserved at $-80^{\circ} \mathrm{C}$. The tissue was homogenized in the buffer solution (0.01 N hydrochloric acid (wako), $1 \mathrm{mM}$ EDTA (sigma), $4 \mathrm{mM}$ sodium disulfite (sigma)), and the supernatant fluid was obtained after centrifugation $\left(15,000 \mathrm{rmp} \times 30\right.$ minutes, $\left.4^{\circ} \mathrm{C}\right)$. Dopamine (Dopamine Research RIA, Labor Diagnostika Nord, Nordhorn, Germany) and serotonin (Serotonin Research RIA, Labor Diagnostika Nord, Nordhorn, Germany) were measured by each ELIZA Kit. The total amount of protein was then measured (BCA Protein Assay kit Pierce, Rockford, IL, USA).

\subsection{Statistical Analysis}

All data were presented as mean \pm S.E.M. The data were analyzed with Student's t-test to evaluate differences between mild-heat stimulation group and the control group. $\mathrm{P}$ values of $<0.05$ were considered as statistically significant.

\section{Results}

\subsection{The Effect of Isolated Rearing on Behavior}

The time spent in the light compartment and the number of transfer between the dark and light compartments in the isolation-stimulation group, the group-stimulation group and the control groups were quantified. The time spent in the light compartment was significantly decreased in the isolation-control group compared with the group-control group (the isolation-control group $144.33 \pm 8.2 \mathrm{sec}(\mathrm{n}=17)$, the group-control group $183.4 \pm 8.4$ sec $(\mathrm{n}=19), p<0.01$; Figure 2(a)). This decrease of the time spent in the light compartment seen in the isolation-control group was significantly increased to the group-control level by peripheral mild-heat stimulation (5 min each, 3 times a week for 2 weeks; the isolation-stimulation group; Figure 2(a)). The time spent in the light compartment in the group-stimulation group was increased compared with the group-control group, but no significant difference was observed (Figure 2(a)).

No significant inter-group changes were observed in the number of transfer between two compartments (Figure 2(b)). The time spent in the light compartment per each transfer was decreased (the isolation-control group $9.4 \pm 0.9 \mathrm{sec}, \mathrm{n}=17$; the isolated-stimulation group $15.1 \pm 2.3 \mathrm{sec}, \mathrm{n}=19, p<0.05$; Figure 2(c)) in the isolation-control group compared with the group-control group, and the peripheral mild-heat stimulation significantly increased this value (the isolation-control group $9.4 \pm 0.9 \mathrm{sec}, \mathrm{n}=17$; the isolated-stimulation group $13.6 \pm$ $1 \mathrm{sec}, \mathrm{n}=20, p<0.01)$. No significant difference was observed between the group-control group and the groupstimulation group.

\subsection{The Change of Corticosterone by Isolated Rearing Stress}

It was found that serum corticosterone was significantly increased at 2 weeks $(1839.2 \pm 115.6 \mathrm{pg} / \mathrm{ml}, \mathrm{n}=9, p<$ $0.001)$, 4 weeks ( $2903.1 \pm 367.9 \mathrm{pg} / \mathrm{ml}, \mathrm{n}=9, p<0.001)$ and 6 weeks $(3647.2 \pm 268.6 \mathrm{pg} / \mathrm{ml}, \mathrm{n}=4, p<0.01)$ after isolation rearing compared with the group-rearing group $(1024.3 \pm 32.4 \mathrm{pg} / \mathrm{ml}$ at 2 weeks, $\mathrm{n}=9 ; 1168.5 \pm$ $57.2 \mathrm{pg} / \mathrm{ml}$ at 4 weeks, $\mathrm{n}=9 ; 1284.3 \pm 57.4 \mathrm{pg} / \mathrm{ml}$ at 6 weeks, $\mathrm{n}=4$; Figure 3$)$. There were also significant differences between 2 weeks and 4 weeks of isolation $(p<0.01)$ and between 2 weeks and 6 weeks of isolation $(p<$ 0.001; Figure 3).

\subsection{The Effect of Mild-Heat Stimulation on Serum Corticosterone}

The increase of serum corticosterone (see Figure 3) by isolated rearing in the isolation group was significantly 


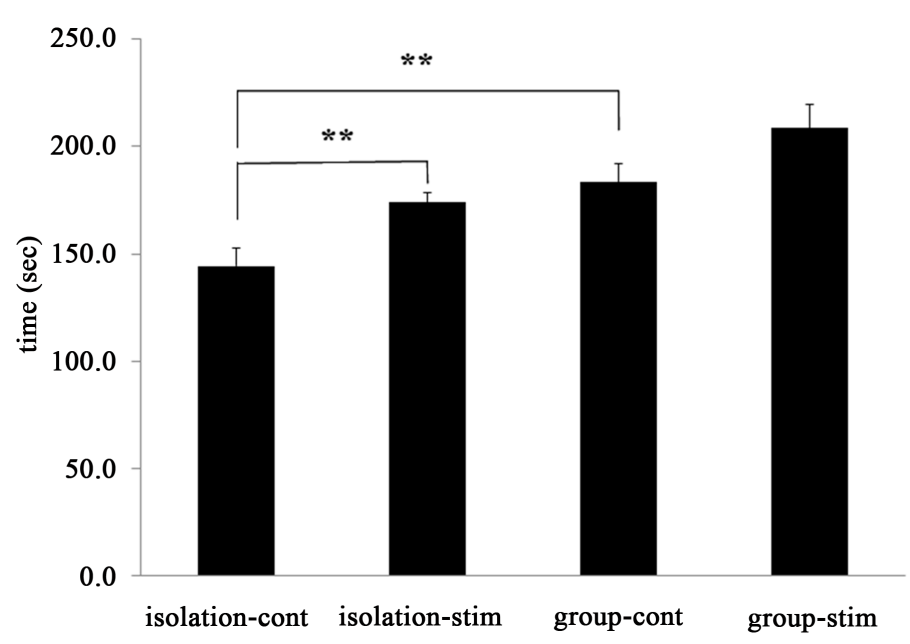

(a)

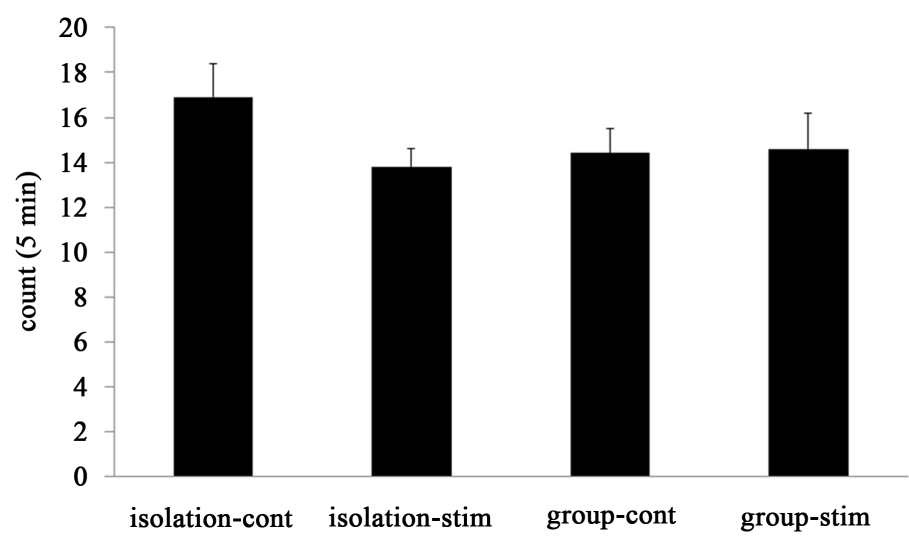

(b)

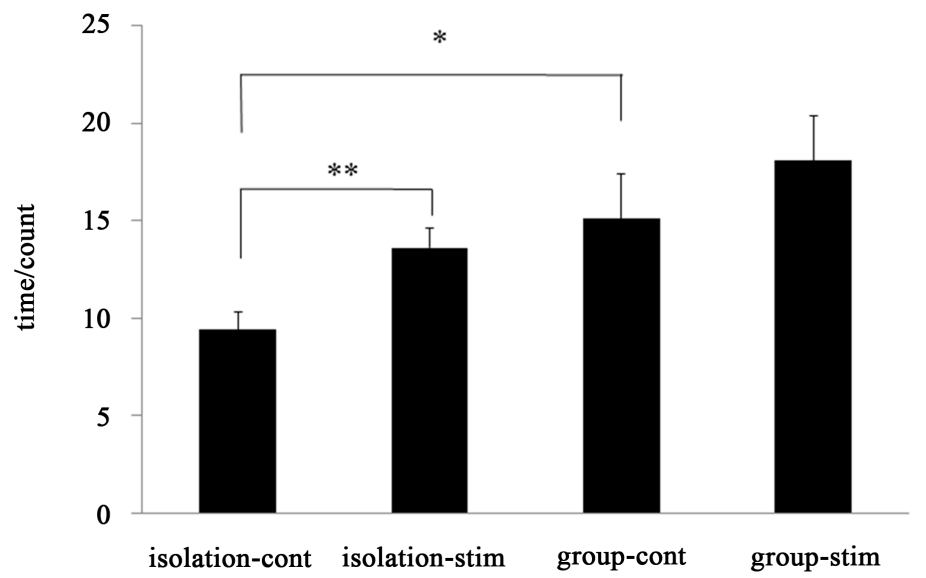

(c)

Figure 2. (a) The 6 weeks social isolation stress significantly decreased the time spent in the light compartment (isolation-cont) of light/dark box, compared with the group-reared control group (group-cont). This decrease was restored to the control level by brief ( $5 \mathrm{~min}$ ) mild-heat stimulation applied during the last 2 weeks (3 times a week) of the 6 weeks stress (isolation-stim). Values are mean \pm S.E.M. ${ }^{* *} p<0.01$ vs. control; (b) The 6 weeks social isolation stress (isolation-cont) did not change the number of transfer between the light and dark compartments compared with the group-reared control group (group-cont). Mild-heat stimulation also did not change the number of transfer. Values are mean \pm S.E.M; (c) The 6 weeks social isolation stress (isolation-cont) significantly decreased the time spent in the light compartment par each transfer, and this value was significantly increased by mild-heat stimulation. Values are mean \pm S.E.M. ${ }^{*} p<0.05,{ }^{* *} p<0.01$. 


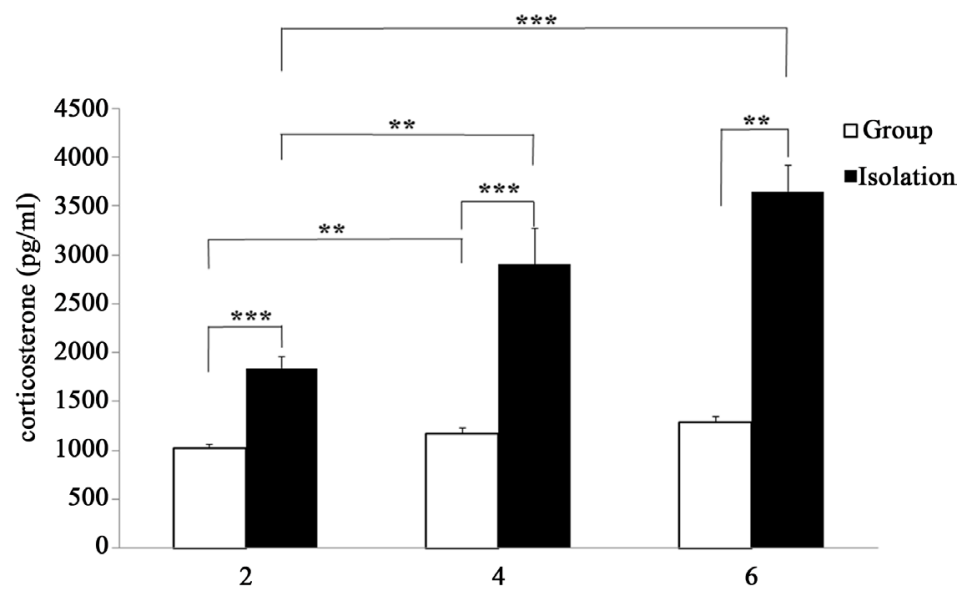

Figure 3. Serum corticosterone levels significantly increased at 2, 4, and 6 weeks after isolated rearing (black bars) compared with the group rearing group (white bars). There were also significant differences in corticosterone levels in the isolation rearing group between 2 and 4 weeks of isolation and between 2 and 6 weeks of isolation. Values are mean \pm S.E.M. ${ }^{*} p<$ $0.05,{ }^{* *} p<0.01,{ }^{* * *} p<0.001$.

decreased by mild-heat stimulation applied during the last 2 weeks of isolation (the isolation-control group $3647.2 \pm 68.6 \mathrm{pg} / \mathrm{ml}, \mathrm{n}=4$; the isolation-stimulation group $1670.4 \pm 126.4 \mathrm{pg} / \mathrm{ml}, \mathrm{n}=4, p<0.01$; Figure 4). No effects of mild-heat stimulation on serum corticosterone level were observed in the group-rearing group (control group, $1284.3 \pm 57.4 \mathrm{pg} / \mathrm{ml}(\mathrm{n}=4)$, stimulation group, $1160.8 \pm 97.8 \mathrm{pg} / \mathrm{ml} \mathrm{n}=5$; Figure 4$)$.

\subsection{The Effect of Mild-Heat Stimulation on Serum Total IgE Level}

The serum total IgE level was significantly increased in the isolation group after 6 weeks of isolated rearing compared with the group-rearing group (the isolation-control group $6.54 \pm 0.52 \mathrm{pg} / \mathrm{ml}, \mathrm{n}=8$; the group-control group $4.00 \pm 0.47 \mathrm{pg} / \mathrm{ml}, \mathrm{n}=9, \mathrm{p}<0.01$; Figure 5 ). This increase was significantly lowered by mild-heat stimulation (the isolation-control group $6.54 \pm 0.52 \mathrm{pg} / \mathrm{ml}, \mathrm{n}=8$, the isolation-stimulation group $3.68 \pm 0.34 \mathrm{pg} / \mathrm{ml}$, $\mathrm{n}=10, p<0.001$ ). In the case of the group-rearing group, the serum total IgE level in the stimulation group was also significantly decreased by mild-heat stimulation (the group-control group $4.00 \pm 0.47 \mathrm{pg} / \mathrm{ml}, \mathrm{n}=9$; the group-stimulation group $2.13 \pm 0.29 \mathrm{pg} / \mathrm{ml}, \mathrm{n}=10, p<0.01$; Figure 5).

\subsection{The Effect of Mild-Heat Stimulation on Serum Interleukin-6}

Serum Interleukin-6 was significantly increased in the isolation group after 6 weeks of isolated rearing compared with the group-control group (the group-control group $106.7 \pm 9.9 \mathrm{pg} / \mathrm{ml}, \mathrm{n}=7$, the isolation-control group $177.1 \pm 6.9 \mathrm{pg} / \mathrm{ml}, \mathrm{n}=6, p<0.001$; Figure 6). This increase was significantly lowered by mild-heat stimulation (the isolation-control group $177.1 \pm 6.9 \mathrm{pg} / \mathrm{ml}, \mathrm{n}=6$, the isolation-stimulation group $148.3 \pm 7.7 \mathrm{pg} / \mathrm{ml}$, $\mathrm{n}=7, p<0.05$; Figure 6). A similar stimulation effect was observed also in the group-rearing groups (the group-control group $106.7 \pm 9.9 \mathrm{pg} / \mathrm{ml}, \mathrm{n}=7$, the group-stimulation group $71.5 \pm 4.5 \mathrm{pg} / \mathrm{ml}, \mathrm{n}=8, p<0.01$; Figure 6).

\subsection{The Effect of Mild-Heat Stimulation on Frontal Cortex Dopamine Level}

There were no significant differences in the amount of dopamine in the frontal cortex between the group-control group and the isolation-control group after 6 weeks of isolated rerating (Figure 7). Mild-heat stimulation did not significantly increase the amount of dopamine in the isolation group, but a significant increase of dopamine by stimulation was observed in the group-rearing group (the group-control group $10 \pm 2 \mathrm{pg} / \mu \mathrm{g}$ protein, $\mathrm{n}=7$, the group-stimulation group $17 \pm 1 \mathrm{pg} / \mu \mathrm{g}$ protein, $\mathrm{n}=7, p<0.05$; Figure 7).

\subsection{The Effect of Mild-Heat Stimulation on Frontal Cortex Serotonin Level}

The amount of serotonin in the frontal cortex was significantly decreased in the isolation group compared with 


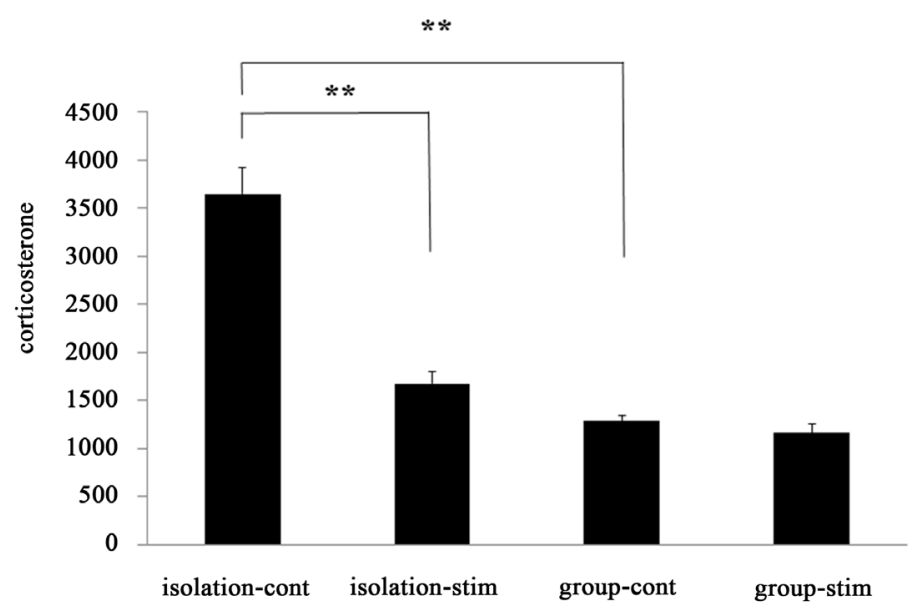

Figure 4. The increased serum corticosterone by 6 weeks social isolation stress (isolation-cont)was decreased to the control level by brief ( $5 \mathrm{~min}$ ) peripheral mild-heat stimulation (3 times a week for 2 weeks; isolation-stim). Mild-heat stimulation did not change the corticosterone value in group-reared mice (group-stim). Values are mean \pm S.E.M. ${ }^{* *} p<0.01$.

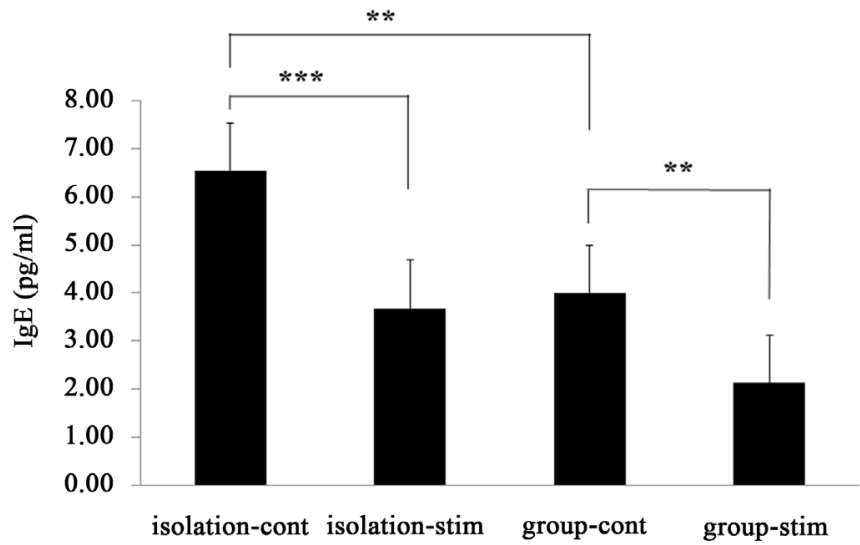

Figure 5. The 6 weeks social isolation stress (isolation-cont) significantly increased the serum IgE level compared with the group-reared control (group-cont), and this increase was lowered to the control level by brief peripheral mild-heat stimulation (isolation-stim). The heat stimulation also significantly decreased the IgE level in the group-reared control mice (groupstim). Values are mean \pm S.E.M. ${ }^{* *} p<0.01 ;{ }^{* * *} p<0.001$.

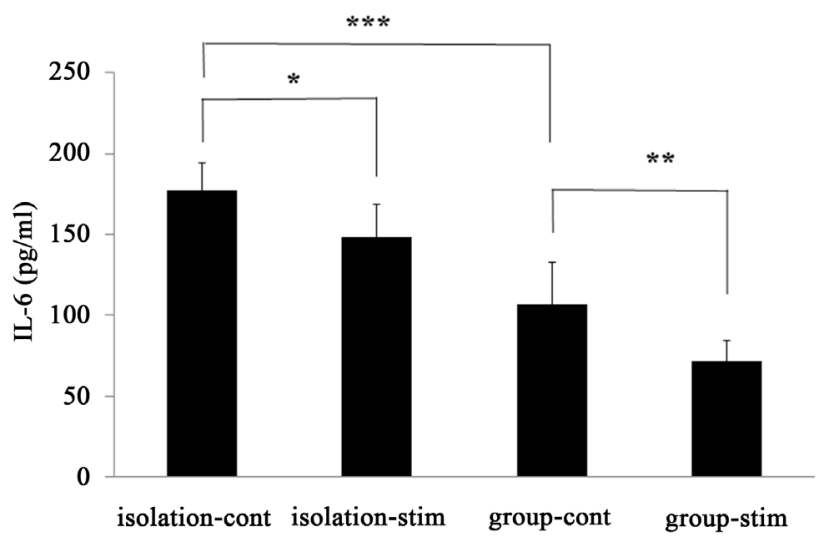

Figure 6. The 6 weeks social isolation stress (isolation-cont) significantly increased the serum IL-6 level compared with the group-reared control (group-cont), and this increase was significantly lowered by brief peripheral mild-heat stimulation (isolation-stim). The mild-heat stimulation also decreased the IL-6 level in the group-reared control mice (group-stim). Values are mean \pm S.E.M. ${ }^{*} p<0.05 ;{ }^{* *} p<0.01 ;{ }^{* * *} p<0.001$. 


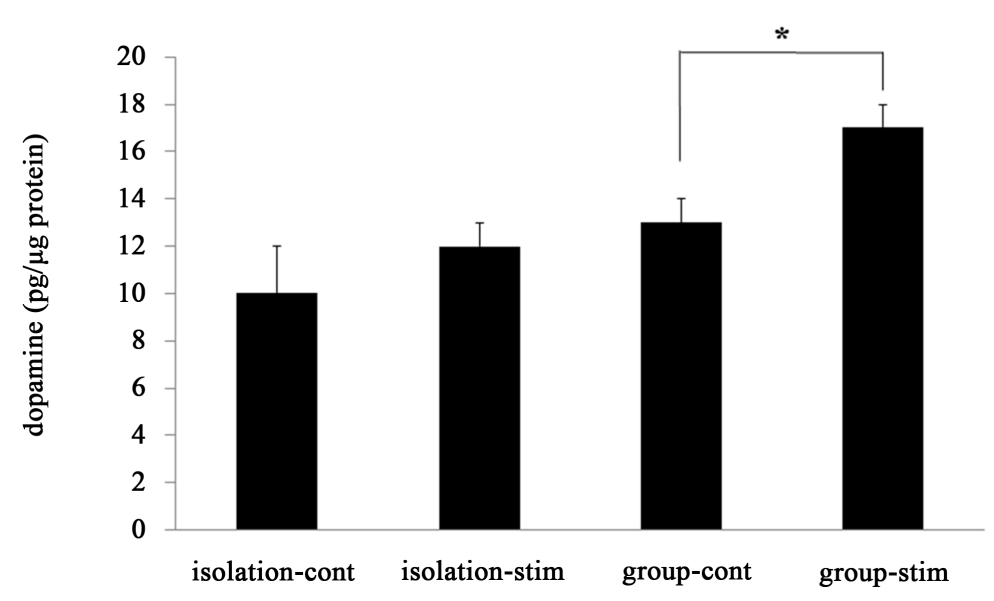

Figure 7. Dopamine content in the frontal cortex did not change after 6 weeks of social isolation stress (isolation-cont) compared with the group-reared control mice (group-cont). Brief peripheral mild-heat stimulation did not change the dopamine level in the stressed mice (isolation-stim), but it significantly increased the dopamine level in the group-reared mice (group-stim). Values are mean \pm S.E.M. ${ }^{*} p<0.05$.

the group-control group after 6 weeks of isolation rearing (the group-control group $25 \pm 2 \mathrm{pg} / \mu \mathrm{g}$ protein, the isolation-control group $17 \pm 2 \mathrm{pg} / \mu \mathrm{g}$ protein, $p<0.01$; Figure 8). This decrease in the isolation group was not significantly improved by mild-heat stimulation, but mild-heat stimulation caused a significant increase of the amount of serotonin in the group-rearing group (the group-control group $25 \pm 2 \mathrm{pg} / \mu \mathrm{g}$ protein, $\mathrm{n}=7$, the groupstimulation group $30 \pm 1 \mathrm{pg} / \mu \mathrm{g}$ protein, $\mathrm{n}=8, p<0.01$; Figure 8 ).

\section{Discussion}

The present results showed that the 6 weeks social isolation stress in mice caused anxiety as indicated by a decreased time spent in the light compartment of the light-dark box and that this anxiety was ameliorated to the control group level by peripheral brief ( $5 \mathrm{~min}$ ) mild-heat stimulation at abdomen and a plantar points, applied 3 times a week for 2 weeks. Moreover, the values of blood corticosterone, IgE and IL-6 were significantly increased by social isolation stress, and these increases were significantly inhibited by the mild-heat stimulation. Finally, while the amount of serotonin in the frontal cortex was significantly decreased by the social isolation stress, mild-heat stimulation had no effect on this serotonin change.

Chronic social stress is known to exert its impact on the body through the neuroendocrine system, autonomic nervous system, chronic inflammation, and oxidant stress [24]. Stimulation by stress is thought to be transmitted to the hypothalamic area through the cortex and the limbic system, and promotes the production of corticotrophin releasing hormone $(\mathrm{CRH})$ in the paraventricular nucleus of hypothalamus; as a result, adrenocorticotropic hormone (ACTH) is released and promotes a production and release of glucocorticoid (the main component of which is cortisol in humans and corticosterone in rodents) from the adrenal cortex [25]. This hypothalamic-pituitary-adrenal (HPA) axis has been implicated for years as a pathological mechanism of mood disorder. For example, it has been indicated since 1960's that the level of cortisol in urine and blood and its metabolites are high in the patients of depression [26] [27]. In the present study, we found that serum corticosterone was indeed significantly increased by isolated rearing compared with the group-rearing control.

The physiological responses including the autonomic nervous system and the HPA axis and the behavioral reactions such as avoidance and escape, both caused by the exposure to stress, are essentially protective responses to the stress [21] [22]. Besides the peripheral effects such as increased blood sugar level, the release of glucocorticoid is known to release dopamine in the prefrontal cortex [28], another example for the adaptive response. However, it is also indicated that the brain system involved in stress reactions is formed dynamically depending on the developing process [29] [30], where stress given in the juvenile stage decreases hippocampal volume [31] and has impacts on emotions and cognitive functions after maturation [32]. Furthermore, a prolonged stress chronically decreases the release of dopamine in the prefrontal cortex and impairs working memory [33]. The results of the present study confirmed that a prolonged isolation stress from the adolescence stage, i.e. 28 days after birth, increased anxiety and declined the amount of serotonin in the frontal cortex. No statisti- 


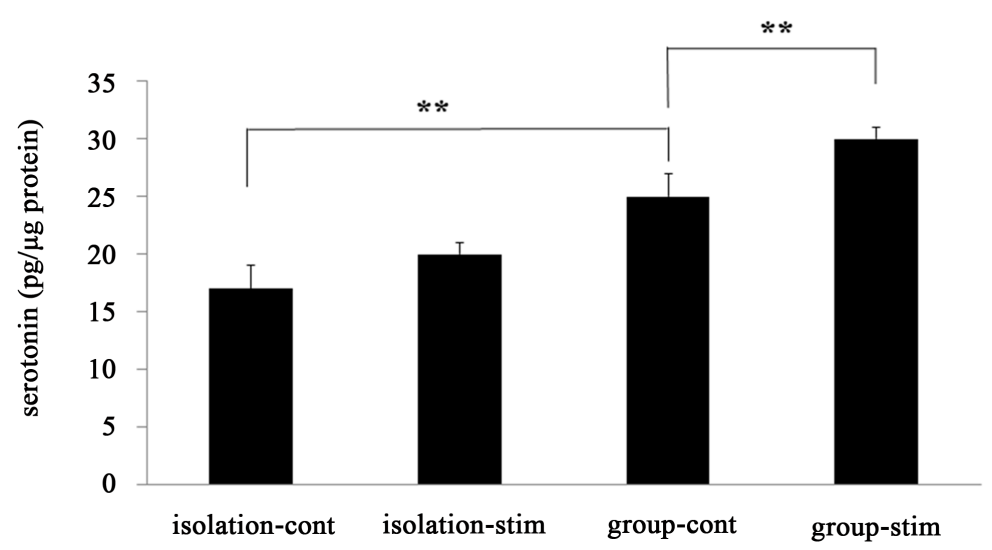

Figure 8. Serotonin content in the frontal cortex decreased significantly by the 6 weeks social isolation stress (isolation-cont) compared with the group-reared control group (group-cont). Brief peripheral mild-heat stimulation did not change the serotonin level in the stressed mice (isolation-stim), but it significantly increased the serotonin level in the group-reared mice (group-stim). Values are mean \pm S.E.M. ${ }^{* *} p<0.01$.

cally significant decrease of the amount of dopamine was observed. However, social isolation stress has been previously shown to decrease dopamine in the prefrontal cortex, as well as serotonin and glutamate [34]. The absence of the difference in dopamine content in the present study may be due to the fact that we dissected the whole frontal cortex rather than the confined, prefrontal area.

It is unknown at present how the prolonged social stress induces decreases of the amount of brain monoamine. But it is possible that the promotion of the production and release of glucocorticoid by stress stimulation is involved. As mentioned above, glucocorticoid receptor stimulation in the prefrontal cortex increases the release of stress-related neuromodulator dopamine [28] in the prefrontal cortex, through the positive-feedback system to midbrain dopamine neurons [35]. The present results demonstrated that serum glucocorticoid was significantly increased 2 weeks after the isolated rearing, and this increase was boosted further after 4 to 6 weeks of stress delivery (Figure 3). Such chronic increases of glucocorticoid may rather induce adapted decreases of dopamine release in the prefrontal cortex. It is also reported that the serotonin release in the prefrontal cortex is decreased by isolation stress [34], thus consistent with the present results, although the underlying mechanisms are still unclear.

Whatever the mechanisms, brain monoamines are involved in controlling emotions and cognitive behaviors, and a decrease of these neuromodulators would result in the abnormality of emotions and cognitive behaviors. As is well known, dopamine neurons project to the limbic and the cortical areas from the ventral tegmental area (VTA), and controls cognition, reward-related learning, and emotional behavior [36]-[38]. Especially, decreased dopamine in the prefrontal cortex results in the dysfunction of this cortex, and this may in turn lead to a decreased inhibition on the amygdala, resulting in the increased anxiety [39]. On the other hand, serotonin, synthesized in the regions including dorsal raphe nucleus, is projected to various areas in the brain including the cortex and the hippocampus [40] [41]. It is thought that a decrease of serotonin is the most important biological factor for causing depression [42], and serotonin is indeed involved in controlling of emotions [43]. It was shown in rodents that the decrease of serotonin synthesis in the brain caused declines of cognitive functions, depression, and anxiety [44]. In the present study, however, peripheral mild-heat stimulation did not restore the amount of serotonin in the frontal cortex, indicating that the anti-anxiety effect of mild-heat stimulation might involve other mechanisms. In the group-rearing group, on the other hand, the peripheral mild-heat stimulation did increase the amount of dopamine and serotonin: thus, the peripheral mild-heat stimulation can up-regulate frontal cortex monoamine levels in non-stressed animals.

We also demonstrated that chronic social stress increases serum IgE and IL-6, and that the increases of these factors are significantly inhibited by peripheral mild-heat stimulation. Enhanced release of glucocorticoid and catecholamine by stress changes the balance of Th1 cells and Th2 cells [45], where Th2 cells become predominant leading to an increase of serum IgE level [46]. It is known that the IgE level increases in the patients with allergic rhinitis, allergic asthma, and allergic dermatitis, and that the IgE value correlates with the severity of patients with allergic rhinitis and allergic dermatitis [46]. It is possible that peripheral mild-heat stimulation de- 
creases corticosterone and inhibits its effect on Th1/Th2 balance; mild-heat stimulation thus may have a function to decrease the risk of developing allergic diseases by inhibiting the amount of serum IgE.

On the other hand, IL-6 is produced by astrocytes and microglia in the brain [47] and is known as an inflammatory marker cytokine for schizophrenia, depression, anxiety disorder, and mood disorders caused by chronic stress [48] [49]. According to the recent findings, IL-6 regulates the activity of ERK (extracellular signal-regulated kinases) which is an important second messenger regulating neuronal plasticity, and IL-6 indeed decreases hippocampal LTP and cortical synaptic transmission [47]. Therefore, the increase of IL-6 level in the prefrontal cortex may decrease the activity of this cortical area, which might result in an increased emotional behavior controlled by the amygdala.

It was previously shown in human subjects that the peripheral mild-heat stimulation decreases blood ACTH and cortisol, and increases the core body temperature and the cerebral blood flow [12]-[14]. The present study extended these results by using the laboratory animals for the first time. Thus, the increased corticosterone level by stress was significantly inhibited by peripheral mild-heat stimulation, and the increases of other stress markers IgE and IL-6 by stress were also significantly countered by peripheral mild-heat stimulation. This novel method is called "Stress Free" for its potential anti-stress effects, and the results of the present study using the mouse model suggest that this peripheral stimulation method indeed may normalize stress-related emotional changes. It remains to be demonstrated now how this peripheral mild-heat stimulation induces these endocrinological and behavioral changes. For example, moderate heat stimulation to specific peripheral sites might exert inhibitory effects on e.g. hypothalamus through afferent inputs.

\section{Conclusion}

In conclusion, chronic social stress is a major problem in our society. Nevertheless, there are no effective methods to date to treat cognitive and emotional abnormalities caused by social stress. The present study demonstrates the possibility that the brief, peripheral mild-heat stimulation alleviates stress-related anxiety. This result may provide a unique contribution to the field of neuropsychiatry.

\section{References}

[1] Chrousos, G.P. (2009) Stress and Disorders of the Stress System. Nature Reviews Endocrinology, 5, 374-381. http://dx.doi.org/10.1038/nrendo.2009.106

[2] Katon, W. and Sullivan, M.D. (1990) Depression and Chronic Medical Illness. The Journal of Clinical Psychiatry, 51, 3-11.

[3] Xie, Y.C. and Li, Y.H. (2009) Observation on Therapeutic Effect of Acupuncture at Zhongwan (CV 12) and Si-Guan Points Combined with Reinforcing-Reducing Manipulation of Respiration for Treatment of Depression. Chinese Acupuncture \&Moxibustion, 29, 521-524.

[4] Zhang, J.B., Ren, L. and Sun, Y. (2009) Meta-Analysis on Acupuncture for Treatment of Depression in Patients of Poststroke. Chinese Acupuncture \& Moxibustion, 29, 599-602.

[5] Ako, N., Tatsuyo, I., Munenori, M. and Yasuzo, K. (1998) A Case of Miscellaneous Complaints Treated by Acupuncture. Journal of the Japan Society of Acupuncture and Moxibustion, 48, 395-401.

[6] Kondo, T. and Kawamoto, M. (2014) Acupuncture and Moxibustion for Stress-Related Disorders. BioPsychoSocial Medicine, 24, 7. http://dx.doi.org/10.1186/1751-0759-8-7

[7] Honma, Y. (1984) Illustration of “The Efficacy of Fourteen Meridians”. Idononihonsha, Yokosuka.

[8] Inoue, K., Aikawa, H. and Yamauchi, T. (2003) Autonomic Nervous Activity and Arousal Level of Patients with Anxiety Disorder (in Japanese). Japanese Journal of Clinical Neurophysiology, 31, 499-502.

[9] Shea, A.K., Kamath, M.V., Fleming, A., Streiner, D.L., Redmond, K. and Steiner, M. (2008) The Effect of Depression on Heart Rate Variability during Pregnancy. A Naturalistic Study. Clinical Autonomic Research, 18, 203-212.

[10] Tonhajzerova, I., Ondrejka, I., Javorka, K., Turianikova, Z., Farsky, I. and Javorka, M. (2010) Cardiac Autonomicregulation Is Impaired in Girls with Major Depression. Progress in Neuro-Psychopharmacology \& Biological Psychiatry, 34, 613-618. http://dx.doi.org/10.1016/j.pnpbp.2010.02.023

[11] Li, Z.P., Qu, S.S., Zhang, J.P., Wu, C.X., Zhang, S.Q., Chen, J.R., Ouyang, H.L., Tang, C.Z. and Huang, Y. (2013) Mechanism Study of Needling Taichong to Treat Hypertension. Journal of Liaoning University of Traditional Chinese Medicine, No. 8, 77-78.

[12] Ryotokuji, K., Ishimaru, K., Kihara, K., Namiki, Y. and Hozumi, N. (2013) Effect of Pinpoint Plantar Long-Wave- 
length Infrared Light Irradiation on Subcutaneous Temperature and Stress Markers. Laser Therapy, 22, 93-102. http://dx.doi.org/10.5978/islsm.13-OR-08

[13] Ryotokuji, K., Ishimaru, K., Kihara, K., Namiki, Y., Nakashima, T. and Otani, S. (2014) Effect of Stress-Free Therapy on Cerebral Blood Flow: Comparisons among Patients with Metabolic Cardiovascular Disease, Healthy Subjects and Placesbo-Treated Subjects. Laser Therapy, 23, 9-12. http://dx.doi.org/10.5978/islsm.14-OR-02

[14] Ryotokuji, K., Ishimaru, K., Kihara, K., Nakajima, T., Otani, S. and Namiki, Y. (2015) Preliminary Results of Highly Localized Plantar Irradiation with Low Incident Levels of Mid-Infrared Energy Which Contributes to the Prevention of Dementia Associated with Underlying Diabetes Mellitus. Laser Therapy, 24, $27-32$. http://dx.doi.org/10.5978/islsm.15-OR-03

[15] Henningsen, P., Zipfel, S. and Herzog, W. (2007) Management of Functional Somatic Syndromes. Lancet, 369, 946955. http://dx.doi.org/10.1016/S0140-6736(07)60159-7

[16] Prins, J.B.,van der Meer, J.W. and Bleijenberg, G. (2006) Chronic Fatigue Syndrome. Lancet, 367, 346-355. http://dx.doi.org/10.1016/S0140-6736(06)68073-2

[17] Mirescu, C., Peters, J.D. and Gould, E. (2004) Early Life Experience Alters Response of Adult Neurogenesis to Stress. Nature Neuroscience, 7, 841-846. http://dx.doi.org/10.1038/nn1290

[18] Marie C.P. and Charles, A.M. (2008) The Long-Term Impact of Stress on Brain Function: From Adaptation to Mental Diseases. Neuroscience \&Biobehavioral Reviews, 32, 1071-1072. http://dx.doi.org/10.1016/j.neubiorev.2008.05.013

[19] Valzelli, L. (1973) The "Isolation Syndrome” in Mice. Psychopharmacologia, 31, 305-320. http://dx.doi.org/10.1007/BF00421275

[20] Ibi, D., Takuma, K., Koike, H., Mizoguchi, H., Tsuritani, K., Kuwahara, Y., Kamei, H., Nagai, T., Yoneda, Y., Nabeshima, T. and Yamada, K. (2008) Social Isolation Rearing-Induced Impairment of the Hippocampal Neurogenesis Is Associated with Deficits in Spatial Memory and Emotion-Related Behaviors in Juvenile Mice. Journal of Neurochemistry, 105, 921-932. http://dx.doi.org/10.1111/j.1471-4159.2007.05207.x

[21] Chrousos, G.P. and Gold, P.W. (1992) The Concepts of Stress and Stress System Disorders. Overview of Physical and Behavioral Homeostasis. Journal of the American Medical Association, 267, 1244-1252. http://dx.doi.org/10.1001/jama.1992.03480090092034

[22] Koob, G.F. (1999) Stress, Corticotropin-Releasing Factor, and Drug Addiction. Annals of the New York Academy of Sciences, 897, 27-45. http://dx.doi.org/10.1111/j.1749-6632.1999.tb07876.x

[23] Tomomi, Y., Kyoko, K. and Osamu, E. (2012) The ddY Mouse: A Model of Postprandial Hypertriglyceridemia in Response to Dietary Fat. Journal of lipid research, 53, 2024-2037. http://dx.doi.org/10.1194/jlr.M023713

[24] Takahiro, K., Noriyuki, S., Toyoshi, I. and Shigenbu, K. (2012) Common Biological Factors between Mood Disorders and Diabet. Experimental Medicine, 30, 2008-2012.

[25] Kubota, M. (1998) Social Defeat and Vasopressin. Japanese Journal of Clinical Psychiatry, 27, 31-36.

[26] Holsboer, F. (2000) The Corticosteroid Receptor Hypothesis of Depression. Neuropsychopharmacology, 23, 477-501. http://dx.doi.org/10.1016/S0893-133X(00)00159-7

[27] Kunugi, H., Ida, I., Owashi, T., Kimura, M., Inoue, Y., Nakagawa, S., Yabana, T., Urushibara, T., Kanai, R., Aihara, M., Yuuki, N., Otsubo, T., Oshima, A., Kudo, K., Inoue, T., Kitaichi, Y., Shirakawa, O., Isogawa, K., Nagayama, H., Kamijima, K., Nanko, S., Kanba, S., Higuchi, T. and Mikuni, M. (2006) Assessment of the Dexamethasone/CRH Test as a State-Dependent Marker for Hypothalamic-Pituitary-Adrenal (HPA) Axis Abnormalities in Major Depressive Episode: A Multicenter Study. Neuropsychopharmacology, 31, 212-220.

[28] Lapiz-Bluhm, M.D. (2014) Impact of Stress on Prefrontal Glutamatergic, Monoaminergic and Cannabinoid Systems. In: Pariante, C.M. and Lapiz-Bluhm, M.D., Eds., Behavioral Neurobiology of Stress-Related Disorders, Current Topics of Behavioral Neuroscience, Vol. 18, Springer-Verlag, Berlin, 45-66. http://dx.doi.org/10.1007/7854_2014_331

[29] Helmeke, C., Ovtscharoff Jr., W., Poeggel, G. and Braun, K. (2001) Juvenile Emotional Experience Alters Synaptic Inputs on Pyramidal Neurons in the Anterior Cingulate Cortex. Cerebral Cortex, 11, 717-727. http://dx.doi.org/10.1093/cercor/11.8.717

[30] Schmidt, M.V., Enthoven, L., van der Mark, M., Levine, S., de Kloet, E.R. and Oitzl, M.S. (2003) The Postnatal Development of the Hypothalamic-Pituitary-Adrenal Axis in the Mouse. International Journal of Developmental Neuroscience, 21, 125-132. http://dx.doi.org/10.1016/S0736-5748(03)00030-3

[31] Huot, R.L., Plotsky, P.M., Lenox, R.H. and McNamara, R.K. (2002) Neonatal Maternal Separation Reduces Hippocampal Mossy Fiber Density in Adult Long Evans Rats. Brain Research, 950, 52-63. http://dx.doi.org/10.1016/S0006-8993(02)02985-2

[32] Huot, R.L., Thrivikraman, K.V., Meaney, M.J. and Plotsky, P.M. (2001) Development of Adult Ethanol Preference and Anxiety as a Consequence of Neonatal Maternal Separation in Long Evans Rats and Reversal with Antidepressant 
Treatment. Psychopharmacology, 158, 366-373. http://dx.doi.org/10.1007/s002130100701

[33] Mizoguchi, K., Yuzurihara, M., Ishige, A., Sasaki, H., Chui, D.H. and Tabira, T. (2000) Chronic Stress Inducesimpairment of Spatial Working Memory Because of Prefrontal Dopaminergic Dysfunction. The Journal of Neuroscience, 20, 1568-1574.

[34] Fone, K.C.F. and Porkess, M.V. (2008) Behavioral and Neurochemical Effects of Post-Weaning Social Isolation in Rodents-Relevance to Developmental Neuropsychiatric Disorders. Neuroscience and Behavioral Reviews, 32, 10871102. http://dx.doi.org/10.1016/j.neubiorev.2008.03.003

[35] Barik, J., Marti, F., Morel, C., Fernandez, S.P., Lanteri, C., Godeheu, G., Tassin, J.P., Mombereau, C., Faure, P. and Tronche, F. (2013) Chronic Stress Triggers Social Aversion via Glucocorticoid Receptor in Dopaminoceptive Neurons. Science, 339, 332-335. http://dx.doi.org/10.1126/science.1226767

[36] Marinelli, M., Rudick, C.N., Hu, X.T. and White, F.J. (2006) Excitability of Dopamine Neurons: Modulation and Physiological Consequences. CNS \& Neurological Disorders Drug Targets, 5, 79-97.

[37] Valenti, O., Lodge, D.J. and Grace, A.A. (2011) Aversive Stimuli Alter Ventral Tegmental Area Dopamine Neuron Activity via a Common Action in the Ventral Hippocampus. Journal of Neuroscience, 31, 4280-4289. http://dx.doi.org/10.1523/JNEUROSCI.5310-10.2011

[38] Dalley, J.W. and Everitt, B.J. (2009) Dopamine Receptors in the Learning, Memory and Drug Reward Circuitry. Seminars in Cell \& Developmental Biology, 20, 403-410. http://dx.doi.org/10.1016/j.semcdb.2009.01.002

[39] Goto, Y., Yang, C.R. and Otani, S. (2010) Functional and Dysfunctional Synaptic Plasticity in Prefrontal Cortex: Roles in Psychiatric Disorders. Biological Psychiatry, 67, 199-207. http://dx.doi.org/10.1016/j.biopsych.2009.08.026

[40] Azmitia, E.C. and Segal, M. (1978) An Autoradiographic Analysis of the Differential Ascending Projections of the Dorsal and Median Raphe Nuclei in the Rat. Journal of Comparative Neurology, 179, 641-667. http://dx.doi.org/10.1002/cne.901790311

[41] Molliver, M.E. (1987) Serotonergic Neuronal Systems: What Their Anatomic Organization Tells Us about Function. Journal of Clinical Psychopharmacology, 7, 3S-23S. http://dx.doi.org/10.1097/00004714-198712001-00002

[42] Jans, L.A., Riedel, W.J., Markus, C.R. and Blokland, A. (2007) Serotonergic Vulnerability and Depression: Assumptions, Experimental Evidence and Implications. Molecular Psychiatry, 12, 522-543. http://dx.doi.org/10.1038/sj.mp.4001920

[43] Young, S.N., Smith, S.E., Pihl, R.O. and Ervin, F.R. (1985) Tryptophan Depletion Causes a Rapid Lowering of Mood in Normal Males. Psychopharmacology, 87, 173-177. http://dx.doi.org/10.1007/BF00431803

[44] Blokland, A., Lieben, C. and Deutz, N.E. (2002) Anxiogenic and Depressive-Like Effects, but No Cognitive Deficits, after Repeated Moderate Tryptophan Depletion in the Rat. Journal of Psychopharmacology, 16, 39-49. http://dx.doi.org/10.1177/026988110201600112

[45] Calcagni, E. and Elenkov, I. (2006) Stress System Activity, Innate and T Helper Cytokines, and Susceptibility to Immune-Related Diseases. Annals of the New York Academy of Sciences, 1069, 62-76. http://dx.doi.org/10.1196/annals.1351.006

[46] Kitagaki, H., Hiyama, H., Kitazawa, T. and Shiohara, T. (2014) Psychological Stress with Long-Standing Allergic Dermatitis Causes Psychodermatological Conditions in Mice. Journal of Investigative Dermatology, 134, 1561-1569. http://dx.doi.org/10.1038/jid.2014.31

[47] Gruol, D.L. (2015) IL-6 Regulation of Synaptic Function in the CNS. Neuropharmacology, 96, 42-54. http://dx.doi.org/10.1016/j.neuropharm.2014.10.023

[48] Anisman, H. (2002) Stress, Immunity, Cytokines and Depression. Acta Neuropsychiatrica, 14, 251-261. http://dx.doi.org/10.1034/j.1601-5215.2002.140601.x

[49] Miller, B.J., Buckley, P., Seabolt, W., Mellor, A. and Kirkpatrick, B. (2011) Meta-Analysis of Cytokine Alterations in Schizophrenia: Clinical Status and Antipsychotic Effects. Biological Psychiatry, 70, 663-671. http://dx.doi.org/10.1016/j.biopsych.2011.04.013 